\title{
Using SONG to probe rapid variability and evolution of starspots
}

\author{
James E. Neff ${ }^{1,4}$, Jon Hakkila ${ }^{1}$, Frank Hill ${ }^{2}$, Jason Jackiewicz ${ }^{3}$, Travis \\ S. Metcalfe ${ }^{4}$, Jørgen Christensen-Dalsgaard ${ }^{4,5}$, Søren Frandsen ${ }^{5}$, \\ Frank Grundahl ${ }^{5}$, Hans Kjeldsen ${ }^{5}$, Uffe Gråe Jørgensen ${ }^{6}$, \\ Per Kjærgaard Rasmussen ${ }^{6}$, and Sheng-Hong $\mathbf{G u}^{7}$ \\ ${ }^{1}$ Dept. of Physics \& Astronomy, College of Charleston, Charleston, SC 29424, USA \\ email: neffj@cofc.edu \\ ${ }^{2}$ National Solar Observatory, Tucson, AZ, 85719, USA \\ ${ }^{3}$ Astronomy Dept., New Mexico State University, Las Cruces, NM 88003, USA \\ ${ }^{4}$ High Altitude Observatory, NCAR, Boulder, CO 80307, USA \\ ${ }^{5}$ Dept. of Physics \& Astronomy, Aarhus University, Ny Munkegade, 8000 Aarhus C, Denmark \\ ${ }^{6}$ Niels Bohr Institute, U. Copenhagen, Juliane Maries Vej 30, DK 2100 Copenhagen, Denmark \\ ${ }^{7}$ Yunnan Astronomical Observatory, Chinese Academy of Sciences, Kunming, China.
}

\begin{abstract}
The Stellar Observations Network Group (SONG) is being developed as a network of 1-meter spectroscopic telescopes designed for and primarily dedicated to asteroseismology. It is patterned after the highly successful GONG project. The Danish prototype telescope will be installed in Tenerife in early 2011. Ultimately we hope to have as many as 8 identical nodes providing continuous high-resolution spectroscopic observations for targets anywhere in the sky. The primary scientific goals of SONG are asteroseismology and the search for Earth-mass exoplanets. The spectroscopic requirements for these programs push the limits of current technology, but the resulting spectrograph design will enable many secondary science programs with less stringent requirements. Doppler imaging of starspots can be accomplished using continuous observations over several stellar rotations using identical instrumentation at each node. It should be possible to observe the evolution of starspot morphology in real-time, for example. We discuss the design and status of the SONG project in general, and we describe how SONG could be used to probe short timescale changes in stellar surface structure.
\end{abstract}

Keywords. Stars: spots, stars: oscillations, stars: magnetic fields, stars: activity, instrumentation: spectrographs

\section{The Stellar Observations Network Group}

Carefully designed, dedicated, long-term ground-based (e.g. GONG, BiSON) and spacebased observations (e.g. SOHO) have enabled a detailed look inside the the Sun using the techniques of helioseismology. Extending these techniques to stars ("asteroseismology") has long been a dream of the stellar astrophysics community (Christensen-Dalsgaard 2002). Thanks to intensive ground-based observing campaigns and, especially, the CoRoT and Kepler missions, that dream is now becoming a reality (Bedding et al. 2010, Chaplin et al. 2010, Gilliland et al. 2010). The past decade has also seen an explosion of interest in detecting and characterizing extrasolar planets.

GONG ("Global Oscillations Network Group") is a network of 6 extremely sensitive and stable velocity imagers located around the Earth to obtain nearly continuous helioseismology observations. It has been in operation for about 15 years. For at least that 
long, there have been efforts to develop a similar network for stellar seismology. At last, such a network is being built (Grundahl et al. 2008)! It is a Danish-led effort now entitled SONG ("Stellar Observations Network Group"). The scientific goals of SONG are (1) to study the internal structure and evolution of stars using asteroseismology, and (2) to search for and characterize planets with masses comparable to the Earth in orbit around other stars. It will accomplish the first of these goals using 1-meter telescopes and extremely stable high-resolution spectrographs with an iodine cell. The second goal will be accomplished using lucky imaging for high-cadence microlensing event monitoring and through radial-velocity studies.

\section{Design capabilities and status of SONG}

The Danish prototype has been fully designed and funded (Grundahl et al. 2009). The 1-meter telescope and observatory enclosure is being built by Astelco Systems, GMBH, with delivery at the site in Tenerife expected around the beginning of 2011. The instrumentation and spectrograph enclosure is being built and integrated in Denmark. A second, virtually identical "node" is being developed in China. Preliminary design work has already been completed, and site surveys are being conducted.

A consortium has been formed in the US to actively seek funding for a third node, to be placed in Hawaii (possibly at HAO's Mauna Loa Solar Observatory). Ultimately, we envision an 8-node network (four telescopes in each hemisphere). But even a 3-node network of identical instruments will enable high-resolution spectra to be obtained continuously over a large fraction of the sky.

The telescope is an alt-az design with one Nasmyth focus occupied by two lucky imaging cameras (using a beam splitter for separate red and visual cameras) designed for microlensing planet searches. The coudé focus feeds a spectrograph in a separate, climatecontrolled enclosure (a modified shipping container on a stable platform). An iodine cell in the pre-slit area is crucial to measure radial velocities with the precision required for asteroseismology, but it can be removed from the beam for normal spectroscopic observations. The baseline detector is an Andor Technology $2 \mathrm{Kx} 2 \mathrm{~K}$ CCD with 13.5 micrometer pixels. With the echelle spectrograph design, this will yield 2-pixel resolution of 120,000 with a 1 arc-second slit. The design is optimized for the wavelength range 4814 to 6774 $\AA$, with full wavelength coverage below $5200 \AA$. The spectrograph design permits the utilization of a larger detector with smaller pixels to achieve greater wavelength coverage and better sampling of the instrumental profile. Much of the data pipeline processing will be accomplished on-site, but the final processing will be performed at the observatory operations center in Denmark. SONG is intended to be an "open-source" project - all drawings and in-house software will be available to the community.

\section{The need for continuous spectroscopy}

The use of Doppler imaging techniques has revealed the surface structure (magnetic and otherwise) of a large number of stars (Berdyugina 2005, Strassmeier 2009). For the most part, these have been based on observations from a single site, with data obtained over several rotations to achieve full phase coverage. Targets are routinely observed for only a few days each observing season. It is very difficult to do otherwise using a single, general purpose facility.

How stable is the structure over several rotations? On what timescales do starspots form, migrate, and decay? These questions are difficult or impossible to answer with an occasional "snapshot" made from a single site. Ideally, we would like to observe a 
star continuously over several rotations for a single Doppler image, and we would like to combine a series of Doppler images to investigate the structure over the relevant evolutionary timescales. These can be used to probe the nature of stellar dynamos by measuring differential rotation, meridional flows, and starspot behavior on evolutionary and stellar cycle timescales (e.g. stellar "butterfly diagrams"). To accomplish all this, we require a network of high-resolution spectrographs (preferably with identical capabilities) widely distributed in longitude.

Between 1989 and 1998, a global consortium (known as MUSICOS) was formed, and tremendous effort was made to arrange for continuous high-resolution spectroscopy using existing facilities (Catala et al. (1993)). Several new spectrographs were built and placed at key facilities. Over the decade, 5 major campaigns including 19 scientific programs were carried out. Each of these campaigns interleaved 3 or more scientific programs that all required continuous spectroscopy. Examples include stellar oscillations (e.g., Kennelly et al. (1996)), stellar activity (e.g. García-Alvarez et al. (2003)), and dynamic circumstellar environments (e.g. Unruh et al. (2004)). MUSICOS demonstrated that obtaining continuous high-resolution spectroscopy using existing sites requires a heroic effort, is limited by inhomogeneous data sets from non-identical facilities, and can only be done on an ad hoc and infrequent basis. Recent campaigns dedicated to asteroseismology (e.g. Arentoft et al. (2008), Bedding et al. (2010)) have been more successful, but they also highlight the need for a dedicated, full-time network.

\section{Using SONG to observe starspots}

The design of $S O N G$ is driven by the precise radial velocity requirements for asteroseismology. But, as MUSICOS demonstrated, the capability of continous high-resolution spectroscopy enables other science. Doppler imaging of starspots is the most obvious. There is a growing overlap of the starspot and stellar oscillation communities, driven by the tremendous data coming from CoRoT and Kepler. Many stars have both oscillations and starspots, which can serve as complimentary probes of their internal structure.

Asteroseismology requires long, uninterrupted observing runs on individual stars using the iodine cell. The network will be dedicated for weeks or even months to a single bright target. During periods of overlapping coverage between sites, or at the beginning and end of each night, individual nodes could be scheduled for other programs. The $S O N G$ network therefore will be capable of interleaving observations over starspot evolutionary timescales. For stars with stable spots and suitable periods, this coverage will be adequate for Doppler imaging. For most stars, however, continuous coverage with the full SONG network for several stellar rotations would be preferable. Short Doppler imaging runs could be scheduled between longer asteroseismology runs, for example.

To obtain such continuous Doppler imaging observations, it is necessary that the stellar activity community become involved in the development of SONG. Currently, the top priority is to garner support and funding for the US node, but there is still an opportunity for this community to provide input into the observing and data analysis strategies. Funding the full 8-node $S O N G$ network is likely to require broad support from the entire stellar astrophysics community. We are planning a workshop (to be held in Charleston, SC during September 2011) to solicit broader community input and support.

\section{Acknowledgements}

For more information see http://astro.phys.au.dk/SONG/ 


\section{References}

Arentoft, T. et al. 2008, Astrophys. J., 687, 1180

Berdyugina, S.V. 2005, Living Reviews in Solar Physics, 2, 8

Bedding, T. R. et al. 2010, Astrophys. J., 713, 935

Catala, C. et al. 1993, Astron. Astrophys., 275, 245

Chaplin, W. J. et al. 2010, Astrophys. J., 713, L169

Cristensen-Dalsgaard 2002, Reviews of Modern Physics, 74, 1073

García-Alvarez, D. et al. 2003, Astron. Astrophys., 397, 285

Gillilland, R. L. et al. 2010, Pub. Astron. Soc. Pac., 122, 131

Grundahl, F., Arentoft, T., Christensen-Dalsgaard, J., Frandsen, S., Kjeldsen, H, \& Rasmussen, P. K. 2008, Journal of Physics Conference Series, 118(1):012041

Grundahl, F., Cristensen-Dalsgaard, J., Kjeldsen, H., Jørgensen, U. G. Arentoft, T., Frandsen, S., \& Rasmussen, P. K. 2009, ASP-CS, eds. Dikpati, M. et al., 416, 579.

Kennelly, E. J. et al. 1996, Astron. Astrophys., 313, 571

Strassmeier, K. G. 2009, Astron. Astrophys. Reviews, 17, 251

Unruh, Y. C. et al. 2004, Mon. Not. Roy. Astron. Soc., 348, 1301 